\title{
A 4-step Tool-Chain for IoT Business Model and Sustainability Recommendations
}

\author{
Corinna Schmitt*, Yves Steiner ${ }^{\dagger}$, Reinhard Herzog ${ }^{\ddagger}$, Nicola Terrenghi ${ }^{\S}$, and Burkhard Stiller ${ }^{\dagger}$ \\ *Universität der Bundeswehr München, Research Institute CODE, Neubiberg, Germany, corinna.schmitt@unibw.de \\ ${ }^{\dagger}$ Communication Systems Group CSG, Department of Informatics IfI, University of Zürich UZH, Zürich, Switzerland, \\ yves.steiner2@uzh.ch and stiller@ifi.uzh.ch, \\ ${ }^{\ddagger}$ Fraunhofer-Institut IOSB, Karlsruhe, Germany, reinhard.herzog@iosb.fraunhofer.de, \\ $\S$ University of Lausanne, Faculty of Business and Economics (HEC), Lausanne, Switzerland, nicola.terrenghi@unil.ch
}

\begin{abstract}
Today many different technologies and products are available for the application field of Internet of Things (IoT) and the development continues. Before launching a new product on the market, a market analysis may be used to develop a specific business model (BM) recommendation. This includes not only the classical analysis according to the BM CANVAS (e.g., stakeholders involved, key resources, revenue streams, and cost), but also the analysis of socio-economic aspects up to the identification of sustainability aspects and measures from a Business-to-Business and Business-to-Customer view. Thus, this paper proposes a generic-applicable 4-step tool-chain combining well-established methods with new ones to develop a strong and valid BM for IoT products including the identification of essential sustainability items. This tool-chain is applied to an IoT scenario EduCampus, which forms as a concrete instantiation of the methodology developed.
\end{abstract}

Index Terms - Tool-chain, Internet of Things (IoT), Business Model (BM), Sustainability

\section{INTRODUCTION}

When bringing new products to the market, it is important to base that on a well-defined business model (BM); for instance, which financial scheme is the most suitable, a license model or an open source model. This is challenging by itself and requires detailed knowledge on the current market situation, especially when planning to enter the IoT market. Besides the market analysis a precise understanding of the product itself is required as well as the customer needs and required items concerning sustainability. Usually a BM development is based on classic approaches such as the BM CANVAS [6], [5] or the Value-Network-Analysis (VNA) [3], [8], [13]. However both techniques do not consider the impact and consequences a new product has (i) on existing settings or products and (ii) to existing workflows.

Thus, this paper introduces a new tool-chain (cf. Fig. 1) combining well-accepted methods (e.g., CANVAS and VNA) with newer methods (e.g., tussle analysis [12] and Terrenghi's method [11]) in order to define a proper BM and sustainability recommendation. It requires a well-defined scenario as initial input. Further input can include individual experiences and detailed technical knowledge. Each step creates specific output that can be used on its own for the individual purpose of the method applied. Within the approach proposed here the

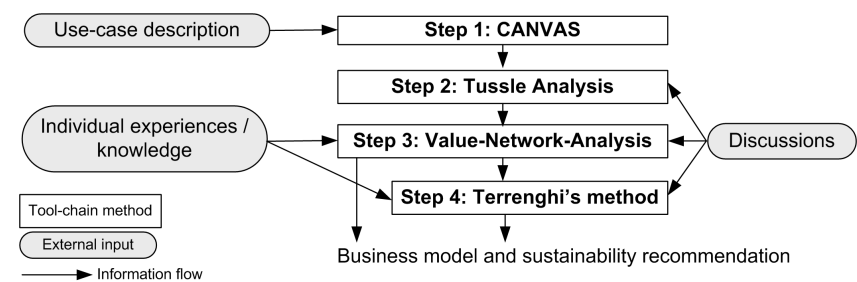

Fig. 1: Proposed 4-step tool-chain concept

output is in return used as input for the next step of the method. Additionally, discussions between developers and target customers need to be included in the process of the tool-chain in order to receive feedback for each step of the process.

In order to show how the tool-chain works and can be applied a specific scenario is essential. The tool-chain was designed within the EU H2020 project symbIoTe [2] having the goal to bring the IoT platform symbIoTe onto the IoT market supporting interoperability. symbIoTe investigates five IoT scenarios [9], [10]. For a proof of concept of the toolchain proposed the scenario EduCampus is selected. It is inspired by the eduroam (EDUcation ROAMing) initiative [1]. The key idea is to agree on a common framework to harmonize infrastructure services, in order to provide researchers, teachers, and students with easy and secure access to campus services when visiting campuses other than their own. While eduroam focuses on the network access, the use-case EduCampus utilizes IoT middleware services such as symbIoTe offers. Looking at the rapidly growing IoT market, applications for sensor and smart devices increase. Thus, the variety of service offerings based on IoT middleware installations will be manifold like access and climate control systems, location and navigation, and room information/booking services on campuses. Sometimes these services will be unique to certain campus, but in many cases there will be very similar services on different campus (e.g., room reservation or access), but implemented in specific deployment manner. This will result in services, which are functionally identical for different campus solutions, but technically incompatible for campus users visiting. In any case there will be a multiplatform deployment 
consisting of different IoT-domains and also of different IoTmiddleware products. By facilitating symbIoTe interoperability for campus deployments, EduCampus can be the incubator for interoperable IoT-platform federations.

Thus, the four methods forming the tool-chain are briefly described. Section III introduces the specific use-case campus federation assumed for the scenario EduCampus as initial input for the tool-chain, followed by applying each step of the tool-chain to them to build a BM recommendation and to identify sustainability items finally. Section IV summarizes and concludes the paper.

\section{TOOL-CHAIN CONCEPT}

When performing a literature research concerning BMs in IoT it can be recognized that many different ones are in place identifying similar items (e.g., stakeholders, customers, revenue streams, costs, or resources). The consent is that for each new product or solution a dedicated BM must be developed. Depending on the use-case assumed two different views must be taken into account during development, namely (i) a Business-to-Customer (B2C) view and (ii) a Businessto-Business (B2B) view. For both views various strategies and tools (e.g., CANVAS, tussle analysis or Value-NetworkAnalysis) are in place, but mainly focusing on one of the views only. Today, both views need to be addressed to develop a promising BM recommendation and to identify relevant short/long-term sustainability items. Thus, the proposed toolchain in this paper combines these two views by starting with $\mathrm{B} 2 \mathrm{C}$ over B2B towards the combined view Businessto-Business-to-Customer (B2B2C).

The proposed tool-chain consists of three commonly known methods applied in a sequence, where the output of the previous method is used as input to the following method. Since the BM shall also address sustainability, a forth method is required. To initiate the tool-chain, a detailed description of the use-case determines a necessity including details of stakeholders involved and targeted, work flows, and resources required. Based on those details the tool-chain is applied to reach a BM recommendation and the identification of sustainability items.

\section{A. CANVAS}

As stated in [6] a BM "is a description of the value a company offers to one or several segments of customers and of the architecture of the firm and its network of partners for creating, marketing, and delivering this value and relationship capital, to generate profitable and sustainable revenue streams."

Osterwald et al. proposed a single reference model known as CANVAS [6], [5]. It includes the most widely used components (also called building blocks) in the BM literature, namely customer segments, value propositions, channels, customer relationships, revenue streams, key resources, key activities, key partnerships, and cost structure. Each of these components answers specific questions to define a BM for a special purpose (e.g., company or project) [6], [5]. A full list is given in [6], [5], [9] and, thus, only some examples are listed:
1) Key partners: Who are our key partners? Who are our key suppliers?

2) Key activities: What key activities do our value propositions, our distribution channels, customer relationships, and revenue streams require?

3) Key resources: What key resources do our value propositions, our distribution channels, customer relationships, and revenue streams require?

4) Revenue streams: For what value are our customers really willing to pay? For what do they currently pay?

All those questions can be addressed by stakeholders related to the product or solution (e.g., the symbIoTe platform). Identified items of those building blocks are used as input for the Tussle Analysis.

\section{B. Tussle Analysis}

Clark et al. [4] were the first to point out the relevance of tussles in the future cyberspace. In a first step, tussle analysis identifies stakeholders and their interests. In a second step, conflicts between these interests and means available to stakeholders to enforce their interests are identified. In the last step, it is investigated, how stakeholders will use their means to enforce their interests and how this can either be prevented or at least ensured that no affected stakeholder suffers from unfair consequences. Such anticipatory evaluation of technology is particularly important, when the technology proposes use-cases that are novel and innovative.The tussles analysis was standardized by an ITU recommendation [12], [7].

\section{Value-Network-Analysis}

Traditional BM approaches like CANVAS highlight processes within the business and show an inside-out perspective on the business or product (e.g., IoT platform). A ValueNetwork-Analysis (VNA) in contrast reflects a global and objective view from the BM ecosystem in general. The goal is to not only obtain the value creation of the business in question, but rather explain relations between stakeholders in the entire value creation process. This is especially crucial for the value creation process of an IoT-Platform, which connects stakeholders with each other and enables to build the valuecreating network, while creating a minimal value itself. In order to map the network of an ecosystem, research suggested to consider stakeholder, actions, and value transactions within the network [3], [8]. In contrast to the traditional BM theory addressing value creation and value capture as the main purpose, [13] defined in the value design approach four aspects that need to be considered in IoT in order to understand how the value is created and captured within an IoT network: (i) Value driver motivating future participants to take part in the entire development and distribution process. (ii) Value nodes are everything bringing value to the IoT system. Classic examples are persons, groups, organizations, and business units that need to be expanded by actions, automated processes, autonomous actors (e.g., programmed systems, learning systems, or smart sensors) for the IoT. 
(iii) Value exchange not only describes how the system works, but also which revenue it creates. (iv) Value extract looks on monetary value (e.g., license fee) created, where the focus is on the extraction of value relevant to own business rather than the entire system.

\section{Terrenghi's Method}

Terrenghi's method [11] seeks information from the three preceding methods and includes especially individual experiences and knowledge complementing the $\mathrm{BM}$ recommendation with sustainability. The main difference of this method here is to start the following process from the customers' perspective: First, define the specific need of a customer (e.g., room booking). Second, identify essential stakeholders, required to reach and fulfill the need. Third, describe interrelationships between stakeholders, where the type of value the stakeholder generates and receives is identified. Forth, describe the benefits of each stakeholder by pointing out perceived advantages. After performing those steps, the sustainability graph can be drawn. As [11] argued, sustainability is a broad term that implies the ability to be maintained at a certain rate or level. Literature adopts this term for multiple contexts and analysis: mainly economics, environmental, and social. Given the intrinsic concept of time in their definition, sustainability needs to have at least two horizons: short- and long-termed; resulting in a sustainability matrix. Those sustainability items identified, allow to optimize the BM intended by answering the leading questions (cf. Section II-A) more specific and support customers' binding to the product by advertising its benefits.

\section{Tool-Chain Applied To Use-CAse}

This tool-chain is applied to the scenario EduCampus to prove its applicability to reach a BM and sustainability recommendations. In order to initiate the tool-chain, a detailed description of the settings assumed are required. EduCampus has two use-cases, namely campus federation and third party catering service. The tool-chain will be applied to the first one. It describes an alliance between different campus providers, by sharing room resources according to a common federation agreement, also known as service level agreement. The main purpose is to provide a better service to campus users, like students searching for working places in partner sites, and at the same time to safe administration costs by simplifying the registration of visiting campus users.

\section{A. Step 1 - CANVAS}

Based on the use-case description (cf. Section III-A) and individual knowledge the guiding questions from the CANVAS cf. Section II-A can be answered by filling the building blocks. Fig. 2 shows the completed CANVAS for the campus federation. ${ }^{1}$

As key partners in CANVAS the symbIoTe consortium, system integrator, campus local administration, and students

\footnotetext{
${ }^{1}$ CANVAS would have similar entries in the building boxes for a third party service case (e.g., catering) and be expanded by items like catering service, advertising, direct sales, or catering costs appearing in the resp. building boxes.
}

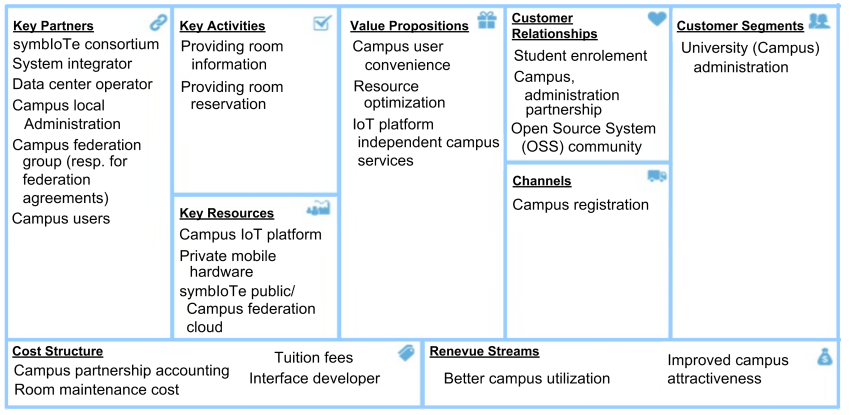

Fig. 2: Filled CANVAS sheet for Campus Federation

using a campus environment were identified. Further key partners are a data center operator, required to maintain all information of participating campuses/universities, and students with required credentials. From the administration point of view, a campus federation group needs to be in place as well being responsible for federation agreements from the legal and finance perspective. The provisioning of room information and room reservation services was identified as key activity that goes hand in hand with the campus' user convenience, the IoT platform independent campus service, and the resource optimization as identified value proposition items. The customer relationship identified here is the Open Source Software (OSS) community. The customer segment is the university (campus) administration. Key resources identified were the campus IoT platform, the private mobile hardware (e.g., smart phone, notebook, tablet), and two clouds (public one and a campus federation one). The latter cloud is required, because a campus-independent resource discovery service is required that should only be available for authorized persons (e.g., students, campus members). With special agreements within a given federation, other deployment strategies are possible, like the hosting of common services within a selected campus-provided data center. The cost structure includes room maintenance costs and a campus partnership accounting, which includes all costs required to maintain the partnership within a campus federation. This may cover administration costs for visiting campus guests, maintenance cost for shared meeting or working place facilities, or compensation allowances for guest tutors and students. The revenue streams achieved contain a better campus utilization and an improved campus attractiveness.

\section{B. Step 2 - Tussle Analysis}

Based on the use-case description and driven by the CANVAS stakeholders' identification (key partnerships and customer segments) the second step from the proposed tool-chain can be performed. First, stakeholder interests must be identified in order to specify possible tussles appearing for this use-case:

1) Campus administration wants to decrease administration costs and increase student satisfaction.

2) Campus users (students) want to decrease their spendings (mainly by tuition fees) and appreciate access to a 
great variety of digital service (e.g., friend finding and room booking via smartphone applications).

3) Campus service providers (e.g., catering service) want to increase their service sales.

4) ID card providers want to increase their ID card sales.

Performing the tussle analysis as described in Section II-B two essential tussles could be identified: (1) The adoption tussle and (2) the location privacy tussle. These two tussles must be addressed first to convince target audience to support and deploy symbIoT's IoT platform. The authors are aware of the fact that other tussles might occur over time and depending on involved new stakeholders hand their wishes (e.g., advertising, payment solution included).

The adoption tussle is generic for the socioeconomic paradigm of the industrialized world, as incompatibilities often increase revenue streams. More precisely, inefficiency often results in the possibility to reach larger gains for selected stakeholder. In this use-case the inefficiency on the students' site is the inability to use a university ID card at different universities. Therefore, if a student moves to another university he/she has to acquire a new card, which is often associated with a fee. This cost may be hidden to students, when the card provider charges the university for the cards provided. The university will cover these costs by tuition fees. Therefore, ID card providers have an interest to maintain this inefficiency, as it allows them to collect more fees. Furthermore, integrating an automated solution will come with extra cost in terms of hiring specialists who integrate this technology. Therefore, ID card providers have no incentive to conduct a costly process that will decrease their ability to sell their services/goods. Therefore, the IoT platform symbIoTe has to be pushed by universities themselves. In particular, the campus administration has to cover the cost for integrating this solution. Also it may be necessary to contract a different ID card provider, if the current one is not willing to adopt the IoT platform symbIoTe. All this implies significant overhead and costs for campus administrations. An advantage that the IoT platform symbIoTe offers to universities is the reduction of administrative overhead. However, this advantage will likely only be sufficient to compensate for the additional overhead and costs in case of campus federations (in this case, students will change campuses frequently). Therefore, such campus federations have to be directly approached by the symbIoTe consortium, to make them aware of and support symbIoTe. Furthermore, students, who are the main beneficiaries of the IoT platform symbIoTe, have to be mobilized to support the adoption. In particular, students directly benefit from the IoT platform symbIoTe by a simplified guest registration and more flexible workplace management. To mobilize students, those benefits have to be clearly demonstrated to them, for example, by giving demonstrations to bodies representing students and their interests.

After the adoption tussle is solved successfully, the IoT platform symbIoTe will provide location-based services, like friend finding or ad-hoc room reservation. However, the stu-

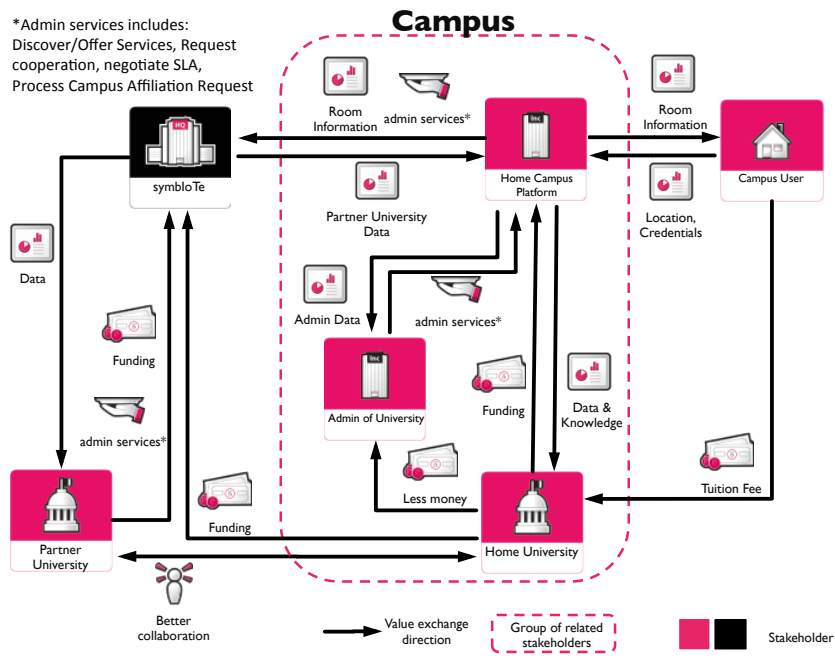

Fig. 3: VNA graph

dent's location (required by these services) is also valuable to campus service providers and other marketeers offering i.e. location-based advertisements, which may degrade the IoT platform symbIoTe to an advertisement platform. To overcome this location privacy tussle, it is important that a student can control who has access to which information he/she provides. For example, a student must have the possibility to grant a friend-finding service access to his/her location, such that he/she can be shown friends in his proximity. At the same time, the student must be able to hide this information from campus service providers, as they may flood the student with push notifications, when he/she gets near their locations.

Having the two tussles identified, implications for the market-release of the IoT platform symbIoTe can be attached to two main activities: (i) Initial investment cost will appear and (ii) the stakeholders must be contacted in the correct order (1. campus administration, 2. campus service providers, 3. campus users) to receive support.

\section{Step 3 - VNA}

As input the stakeholder list from the CANVAS and their interests identified by the tussle analysis are used, as well as known workflows from the use-case description, from discussions, and from individual experiences. All this information leads to the specific characterization and relationships of stakeholders for the VNA visualized in the VNA graph (cf. Fig. 3):

- The intermediate platform symbIoTe connects different universities and campuses within universities.

- A campus user is a person eligible to use the campus network and infrastructure, i.e. a student of this university, a guest student, a professor, a guest professor or any kind of member of a federated partner.

- The home campus platform is an IT-platform serving as an interface for symbIoTe of the university providing the campus services to students, professors, and employees. 
- The university administration handles all back office requests and tasks, which include access authorization and setting up contracts.

- The home university resembles the head of the home university or university board handling all financial aspect and taking decisions.

- The partner university resembles the partner university as a whole with all actors included in the home campus.

The VNA performed distinguishes between a home university and a partner university, where former is shown in a higher granularity to reflect the value network within one university campus. The actors involved in one campus are home campus platform, university's administration, and home university. Within the campus the administration of university provides administration services via the home campus system to the whole network and its campus users, where these include discover and/or offer services, request cooperation, negotiate Service-Level-Agreements (SLA), process campus affiliation requests, and authenticate user access requests. In return they receive the necessary data to perform their services. The home campus platform is funded by the home university itself and provides data and knowledge on the campus to the university. For the home university the effort for campus user access verification from partner universities as well as from other campus within the same university is reduced. Furthermore, the effort to access other collaborative services between two universities, which previously had to be handled individually by the administration, decreases, due to a common interface. Thus, the university reduces spending on its administration. The home university buys sensors and devices from thirdparty infrastructure providers and integrates them into the campus platform. These gather and provide information about the rooms like room temperature or beacons for localizing the campus user. The home campus platform forwards administration services and room information to the symbIoTe platform, which provides the data to the partner university. The home campus platform in return receives the data and services from the partner university via symbIoTe. The two universities benefit further from a better collaboration between each other. The partner universities fund their share of the IoT platform symbIoTe. The campus user pays his/her tuition fee to the home university and receives, after sharing his location and credentials, access to the home campus platform. The platform shares information about the home university as well as the partner university with the user. The campus user will always connect to the home campus platform independent from its location, home campus, or at the partner university.

This processes described will ultimately result in less administrative work and costs by a simplified registration and campus access process for visiting campus user. Providing access to administration services for home-based as well as for visiting campus user, reduces administration effort and cost further.

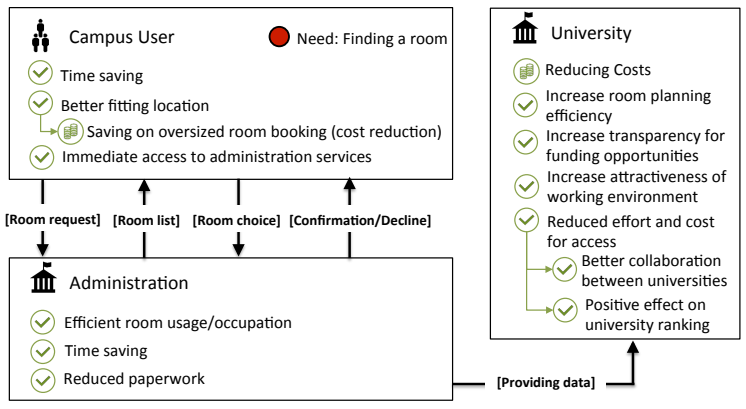

Fig. 4: Sustainability graph

\section{Step 4 - Terrenghi's Method}

After performing the first three steps of the proposed toolchain a BM recommendation is possible, but not in full. Therefore, investigations toward sustainability are required, which is the Terrenghi's method [11] that uses the input used before as well as the output received from each step. Most important are the detailed discussion and the knowledge of the workflows. The best output is received when the scenario is broken down to specific needs in the 2. step of the method (cf. Section II-D). While assuming the "booking a room" setting, the resulting sustainability graph is shown in Fig. 4.

This graph includes sustainability items (green circles) identified mainly from the motivation of stakeholders involved. These can further be categorized into short- and long-term items and mapped to economic, social, and ecological areas filling the sustainability matrix (cf. Tab. I). The authors applied Terrenghi's method to other settings (e.g., catering and navigation) as well and similar sustainability items where identified.

\section{E. Resulting BM and Sustainability Recommendation}

Having the tool-chain performed it can be stated that campus federation support is essential in todays life at universities. The tool-chain delivers a very detailed view on business aspects, involved stakeholders and their needs, resources and activities required, as well as impacts caused by the solution for the use-case. Having all these outcomes in place a prioritization of the financial schemes - licensing or open source model - for a BM recommendation can be done. Which one will be chosen depends highly on if one university takes the lead.

If yes, the recommended model with a business for profit would be a licensing approach. The leading university needs to host and maintain symbIoTe or develop the platform further in the future. In return it receives a license fee from each university joining the network as well as from the service providers (e.g., catering company) for accessing the platform. This model would be close to a commercial business model, which would be an incentive for the operating university to host it depending on how it's priced. Beneficial for the system would be that there is just one organization and one team responsible for symbIoTe and they know the needs of its campus users and other stakeholders from university. 
TABLE I: Sustainability matrix

\begin{tabular}{lll}
\hline & Short-term & Long-term \\
\hline Economical & - Time saving & - Efficient room usage and \\
& - Better fitting location & occupation \\
& - Direct access to customer & - Reducing costs \\
& - Easier way of service offering & - Increase transparency for \\
& & funding opportunities \\
& & - Easier way of offering \\
& & new services \\
\hline Social & - Time saving & - Increase attractiveness of \\
& - Better fitting location & work environment \\
& - Immediate access to & - Reduced effort and cost for \\
& administration and & access \\
& third-party services & - Improve image \\
\hline Ecological & - Better fitting location & - Increase room planning \\
& - Reduced paperwork & efficiency \\
\hline
\end{tabular}

They could as well steer the development of symbIoTe for a long-term satisfaction of all stakeholders.

If none university wants to take the responsibility an open source strategy would be possible as well. Each university joining the network would be responsible for hosting their own symbIoTe setup and keeping it up to date. A committee with members of each contributing partner university should be formed to make decisions on the systems development. Service providers could register and offer their services for free or for an access fee directly to the university on the platform. The costs for operating the platform on their infrastructure would have to be covered by each university itself. In order to join the network federated universities have to accept a SLA in order to participate in the network, independent of which business model is in place. Policy, Responsibilities, Security Issues as well as financial matters are defined in the SLA. In case of the license fee universities have to pay individual fees by either number of user's, monthly subscription or pay-by-usage and should cover the maintenance costs. If it is an open source model required non-monetary contribution to the network and community have to be defined in the agreement as well.

The sustainability matrix shows well-defined items that match the targeted value propositions and revenue streams identified within CANVAS.

\section{SUMMARY}

This paper proposed a newly integrated 4-step tool-chain to develop for a given use-case and IoT technology or platform a well defined BM recommendation, while identifying at the same time essential items of sustainability to satisfy customer needs and to position a product successfully on the market. The tool-chain is defined by using known and new methods. As shown throughout Section III, each step of the tool-chain uses specific input and output from a previous methodology and step. The tool-chain is generic making it applicable for any scenario. The final output - BM and sustainability recommendation - is concrete and resembles specificy when the inputs for each step of the tool-chain is precise and detailed. Individual steps of the tool-chain may undergo several iterations in order to optimize the outcome and, thus, to reach more precise input for the next step. As the tool-chain was applied to the EduCampus use-case successfully, further scenarios will be applied to reach accurate recommendations. The tool chain was also applied to the remaining four scenarios and additional use-cases of symbIoTe showing similar results [9], [10].

The presented results showed that combining well established methods with new ones allow to develop a strong and valid BM for IoT products including the identification of essential sustainability items. The better the scenario is specified, the more meaningful the BM becomes and the more promising the market launch will be. It is recommended to repeat the 4-step tool-chain from time to time in order to react on market changes more quickly. Applying the 4-step tool chain costs time, but gives a perfect visualization of the current situation and what is envisioned and, thus, helps to identify items to further invest on to convince investors successfully.

\section{ACKNOWLEDGEMENT}

This work was supported partially by the symbIoTe project [2], funded by the European Union's Horizon 2020 research and innovation program under grant agreement No. 688156. The work performed by Corinna Schmitt was with UZH.

\section{REFERENCES}

[1] "eduroam," June 14 2018. [Online]. Available: https://www.eduroam.org/

[2] "symbIoTe - symbiosis of smart objects across IoT environments," June 11, 2018. [Online]. Available: https://www.symbiote-h2020.eu/

[3] C. Battistella, K. Colucci, A. F. De Toni, and F. Nonino, "Methodology of Business Ecosystems Network Analysis: A Case Study in Telecom Italia Future Capture," in Technological Forecasting and Social Change, vol. 80, no. 6. New York, NY, USA: Elsevier, 2013, pp. 1194-1210.

[4] D. D. Clark, J. Wroclawski, K. R. Sollins, and S. Braden, "Tussle in Cyberspace: Defining Tomorrow's Internet," in IEEE/ACM Transactions on Networking, vol. 13, no. 3, New York, NY, USA, June 2005, pp. 462 475 .

[5] A. Osterwalder, "The Business Model Ontology - A Proposition In A Design Science Approach," Ph.D. dissertation, University of Lausanne, Lausanne, Switzerland, January 2004.

[6] A. Osterwalder, Y. Pigneur, and C. L. Tucci, "Clarifying Business Models: Origins, Present, and Future of the Concept," in Communications of the Association of Information Systems, vol. 16, no. 1, Atlanta, GA, USA, July 2005, pp. 1-28.

[7] P. Poullie, C. Schmitt, and B. Stiller, "Designing Future Networks: The Investigation of Socio-economic Awareness by the Tussle Analysis," in IEEE Conference on Standards for Communications and Networking, New York, NY, USA, October 2015, pp. 19-24.

[8] P. Ritala, V. Agouridas, D. Assimakopoulos, and O. Gies, "Value creation and Capture Mechanisms in Innovation Ecosystems: A Comparative Case Study," in International Journal of Technology Management, vol. 63, no. 3-4. Geneva, Switzerland: Geneva-Aeroport: Inderscience Enterprises Ltd, 2013, pp. 244-267.

[9] C. Schmitt, P. Poullie, Y. Steiner, and B. Stiller, "symbIoTe Deliverable D1.5 - Final Report on Business Models and Sustainability," https:// tinyurl.com/deliverable15, pp. 1-114, July 2018 (last access: Aug. 29, 2018).

[10] Y. Steiner, "Value-Network-Analysis for IoT Platforms," Master's thesis, Communication Systems Group, Department of Informatics, University of Zurich, Zurich, Switzerland, August 2018.

[11] N. Terrenghi, J. Schwarz, and C. Legner, "Towards Design Elements to Represent Business Models for Cyber Physical Systems," in 26th European Conference on Information Systems, Portsmouth, UK, June 2018, pp. 1-16.

[12] M. Waldburger, P. Poullie, C. Schmitt, and B. Stiller, "Y.3013: Socioeconomic Assessment of Future Networks by Tussle Analysis," ITU-T Telecommunication Standardization Sector of ITU, Geneva, Switzerland, Recommendation Y.3013, August 2014.

[13] M. Westerlund, S. Leminen, and M. Rajahonka, "Designing Business Models for the Internet of Things," in Technology Innovation Management Review, vol. 4, no. 7, Ottawa, Ontario, Canada, July 2014, pp. $5-14$. 